\title{
ARCS DEFINED BY ONE-PARAMETER SEMIGROUPS OF OPERATORS IN BANACH SPACES WITH THE RADON-NIKODYM PROPERTY
}

\author{
CHARLES L. BYRNE
}

ABSTRACT. It is shown that a recent theorem of Junghenn and Taam concerning the domain of the infinitesimal generator of a strongly continuous one-parameter semigroup of operators on a reflexive, locally convex topological vector space remains valid if the domain of the operators is a Banach space with the Radon-Nikodym property. A partial result is obtained for general Banach spaces.

The following theorem is proved in a recent paper by Junghenn and Taam:

Theorem. Let $X$ be a reflexive, locally convex topological vector space. Let $T(t)$, for $t \geq 0$, be a strongly continuous semigroup of operators on $X$, such that for all $c>0, T(c)$ is an isomorphism (into), and let $A$ be the infinitesimal generator of the semigroup. The following are equivalent:

(1) $x$ is in the domain of $A$;

(2) $T() x$ is absolutely continuous on the interval $[0, c]$ for any $c>0$;

(3) $T($ ) $x$ is of bounded variation on the interval $[0, c]$ for any $c>0$.

In the absence of reflexivity, each condition implies the next. It is the purpose of this note to show that if $X$ is a Banach space, then (2) and (3) are equivalent, and if, in addition, $X$ has the Radon-Nikodym property, then all three are equivalent. Among spaces with the Radon-Nikodym property are reflexive Banach spaces and separable dual Banach spaces. For a discussion of the Radon-Nikodym property, see the paper of Rieffel [2]. Recent results characterizing Banach spaces with this property are to be found in [4], [5], and [6].

To prove the above assertions we need two lemmas. Our notation is that of [1]. Terms not defined in this note are as used in that paper.

Lemma 1. Let $X$ be a Banach space, and $x \in X$, for which (3) holds. If $c>0$ and $L$ is the total variation of $T() x$ on $[0, c]$, then $\|T(c+h) x-T(c) x\| / h \leq K L /(c-b)$ for all $0<b<c$, where $K$ is a constant not dependent upon the choice of $h$ (but dependent upon c).

Received by the editors October 4, 1974.

AMS (MOS) subject classifications (1970). Primary 46A05, 47D05; Secondary $46 \mathrm{~A} 25$. 
Prool. Choose $n$ so that $n h \leq c<(n+1) h$, and let $1 \leq j \leq n$. Pick $K$ so that $\|T(t) x\| \leq K\|x\|$ for $t$ in $[0, c]$. Then

$$
\begin{aligned}
\|T(c+h) x-T(c) x\| & =\|T(c+h-j h)\{T(j h) x-T(j h-h) x\}\| \\
& \leq K\|T(j h) x-T(j h-b) x\| .
\end{aligned}
$$

Therefore

$$
n\|T(c+h) x-T(c) x\| \leq \sum_{1}^{n} K\|T(j h) x-T(j h-b) x\| \leq K L .
$$

The assertion follows since we have $c-b<n h$.

Lemma 2. Lnder the same assumptions as Lemma 1, the set $\{\|T(h) x-x\| / h \mid 0<h \leq c\}$ is bounded.

Proof. We have

$$
\|T(h) x-x\| / h=\left\|T(c)^{-1}\{T(c+h) x-T(c) x\}\right\| / h \leq M\|T(c+h) x-T(c) x\| / h,
$$

for $M=\left\|T(c)^{-1}\right\|$. Then $\|T(h) x-x\| / h \leq M K L /(c-h)$, and if $0<b<c / 2$ we have $\|T(h) x-x\| / h \leq 2 M K L / c=Q$. Clearly, for $h$ in $[c / 2, c]$, the numbers are bounded.

We prove now that (3) implies (2) in any Banach space. Let $e>0$ be given and let $\left(a_{i}, b_{i}\right), i=1,2, \ldots, n$, be any collection of nonoverlapping intervals in $[0, c]$. If we first assume that $b_{i}-a_{i}<c / 2$, then

$$
\left\|T\left(b_{i}\right) x-T\left(a_{i}\right) x\right\|=\left\|T\left(a_{i}\right)\left\{T\left(b_{i}-a_{i}\right) x-x\right\}\right\| \leq K\left\|T\left(b_{i}-a_{i}\right) x-x\right\| .
$$

Therefore the choice of $d<e / Q K$ gives $\Sigma_{1}^{n}\left\|T\left(b_{i}\right) x-T\left(a_{i}\right) x\right\|<e$ if $\Sigma_{1}^{n}\left(b_{i}-a_{i}\right)<d$.

Banach spaces $X$ with the Radon-Nikodym property are precisely those spaces with the property that every function of bounded variation from the real line into $X$ is differentiable almost everywhere. This is a classical result due to Bochner and Taylor [3]. If we assume that $X$ has this property, then there is a $\bar{t}>0$ at which point the function $T() x$ is differentiable, if (3) holds. Then, as $h$ approaches $0,[T(\bar{t}+h) x-T(\bar{t}) x] / h$ approaches a limit. It follows that $[T(h) x-x] / h$ does also, since it is equal to $\left[T(\bar{t})^{-1}\left\{\left\{T(\bar{t}+h)_{x}-T(T) x\right\} / h\right\}\right]$. Therefore, if $X$ is assumed to have the Radon-Nikodym property, the three conditions given in the Theorem are equivalent.

\section{REFERENCES}

1. H. Junghenn and C. T. Taam, Arcs defined by one-parameter semigroups of operators, Proc. Amer. Math. Soc. 44 (1974), 113-120.

2. M. A. Rieffel, The Radon-Nikodym theorem for the Bochner integral, Trans. Amer. Math. Soc. 131 (1968), 466-487. MR 36 \#5297. 
3. S. Bochner and A. E. Taylor, Linear functionals on certain spaces of abstractly-valued functions, Ann. of Math. (2) 39 (1938), 913-944.

4. W. J. Davis and R. R. Phelps, The Radon-Nikodym property and dentable subsets in Banach spaces, Proc. Amer. Math. Soc. 45 (1974), 119-122.

5. R. E. Huff, Dentability and the Radon-Nikodym property, Duke Math. J. 41 (1974), 111-114.

6. R. R. Phelps, Dentability and extreme points in Banach spaces, J. Functional Analysis $17(1974), 78-90$.

DEPARTMENT OF MATHEMATICS, THE CATHOLIC UNIVERSITY OF AMERICA, WASHINGTON, D.C. 20064 